\title{
环交联聚磷腈包覆羟基锡酸锶杂化纳米棒 的合成及阻燃环氧树脂研究
}

\author{
张 冲 ${ }^{1}$, 耿晓维 ${ }^{1}$, 高香迪 ${ }^{1}$, 张 欣 ${ }^{1}$, 郭 娆 ${ }^{1}$, \\ 王宇静 ${ }^{1}$, 徐建中 ${ }^{1}$, 马海云 ${ }^{1,2}$
}

(河北大学 1. 化学与环境科学学院; 2. 河北省分析科学技术重点实验室, 保定 071002)

摘 要: 为了开发新型无机-有机杂化的阻燃消烟剂, 本研究采用共沉淀法合成了棒状纳米着基锡酸锶 $\left(\mathrm{SrSn}(\mathrm{OH})_{6}\right)$,

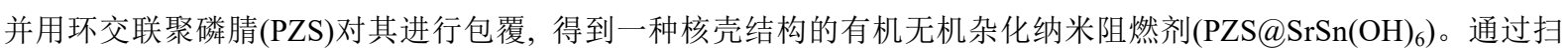
描电镜、透射电镜和红外光谱研究了 $\mathrm{PZS} @ \mathrm{SrSn}(\mathrm{OH})_{6}$ 的微观形貌和化学结构。通过热重分析研究了 $\mathrm{PZS} @ \operatorname{SrSn}(\mathrm{OH})_{6}$ 及 $\mathrm{EP} / \mathrm{PZS} @ \mathrm{SrSn}(\mathrm{OH})_{6}$ 阻燃复合材料的热降解行为。采用极限氧指数和雉形量热对复合材料阻燃 性能进行测试，用 $X$ 射线衍射、扫描电镜、能谱分析及红外光谱对 $\mathrm{EP} / \mathrm{PZS} @ \operatorname{SrSn}(\mathrm{OH})_{6}$ 的阻燃机理进行分析。结 果表明 PZS@ $\mathrm{SrSn}(\mathrm{OH})_{6}$ 在环氧树脂中展现出高阻燃效率和抑烟效果, 且 PZS 与 $\mathrm{SrSn}(\mathrm{OH})_{6}$ 之间存在显著的协同阻 燃效应。与纯环氧树脂相比, 仅添加 $3 \mathrm{wt} \%$ 的 PZS@ $\mathrm{SrSn}(\mathrm{OH})_{6}$ 时, 极限氧指数(LOI)值从 26.2\%增加到 29.6\%。锥形 量热结果表明热释放速率峰值降低了约 $29 \%$, 烟释放速率峰值降低了约 $37 \%$, 残炭率提高了 $242 \%$ 。 PZS@ $S r S n(O H)_{6}$ 在高温下形成致密结构炭层, 隔绝分解产物及热量和氧气交换, 从而显著提高环氧树脂的阻然效果。

关 键 词: 磷腈; 羟基锡酸锶; 杂化; 阻然; 纳米复合材料

中图分类号: 0631 文献标识码: A

\section{Strontium Hydroxystannate Nanorods Encapsulated by Hybrid Polyphosphazene: Synthesis and Flame Retardancy on Epoxy Resin}

\author{
ZHANG Chong ${ }^{1}$, GENG Xiao-Wei ${ }^{1}$, GAO Xiang-Di ${ }^{1}$, ZHANG Xin ${ }^{1}$, GUO Rao ${ }^{1}$, \\ WANG Yu-Jing $^{1}$, XU Jian-Zhong ${ }^{1}$, MA Hai-Yun ${ }^{1,2}$
}

(1. School of Chemistry and Environmental Sciences, Hebei University, Baoding 071002, China; 2. Key Laboratory of Analytical Science and Technology of Hebei Province, Hebei University, Baoding 071002, China)

\begin{abstract}
To develop a novel hybrid organic-inorganic flame retardant and smoke suppressant, strontium hydroxystannate $\left(\mathrm{SrSn}(\mathrm{OH})_{6}\right)$ nanorods were synthesized via a co-precipitation method, and then the $\operatorname{SrSn}(\mathrm{OH})_{6}$ were encapsulated by cyclomatrixpolyphosphazene (PZS) to prepare a novel core-shell organic-inorganic hybrid nano-flame retardant (PZS@SrSn $\left.(\mathrm{OH})_{6}\right)$. The micromorphology and chemical structure were analyzed by scanning electron microscopy (SEM), transmission electron microscopy (TEM) and fourier transform infrared spectroscopy (FT-IR). The thermal degradation of PZS@SrSn $(\mathrm{OH})_{6}$ and EP/PZS@SrSn $(\mathrm{OH})_{6}$ composites was investigated by thermogravimetry analysis (TGA). The flame retardancy properties were studied by limited oxygen index (LOI) and CONE calorimetry tests. The flame retardant mechanism was determined by X-ray diffraction (XRD), SEM, energy-dispersive X-ray spectroscopy (EDS), and FT-IR.
\end{abstract}

收稿日期: 2018-10-17; 收到修改稿日期: 2018-12-27

基金项目: 国家自然科学基金(21306035, 21276059); 河北省基础研究计划(16961402D)

National Natural Science Foundation of China (21306035, 21276059); Basic Research Project of Hebei Province $(16961402 \mathrm{D})$

作者简介: 张 冲(1991-), 男, 硕士. E-mail: hbzc728@163.com

通讯作者：马海云，副教授. E-mail: coffee1123@126.com 
The results showed that PZS@SrSn $(\mathrm{OH})_{6}$ exhibited high flame retardant and smoke suppression efficiency. Significant synergy between PZS and $\mathrm{SrSn}(\mathrm{OH})_{6}$ was found to enhance the flame retardancy of EP compared with pure EP, the LOI value was increased from 26.2\% to 29.6\% with 3wt\% addition of $\mathrm{PZS} @ \mathrm{SrSn}(\mathrm{OH})_{6}$. CONE calorimetry tests indicated that 3\% incorporation of PZS@SrSn $(\mathrm{OH})_{6}$ brought about 29\% and 37\% maximum reduction in peak heat release rate and peak smoke production rate, and $242 \%$ improvement of char residue, respectively. A dense char structure is formed after combustion under elevated temperature for PZS@SrSn $(\mathrm{OH})_{6}$, and the char layer blocks the exchange between the decomposed fragments and oxygen, then protect EP matrix and improve the flame retardancy.

Key words: polyphosphazene; strontium hydroxystannate; hybrid; flame retardancy; nanocomposites

环氧树脂因其优异的耐热性、良好的力学性能 和化学稳定性而被广泛应用于电子、建筑、胶粘剂、 航空等众多领域 ${ }^{[1-3]}$, 但由于其易燃性, 对其进行阻 燃改性一直是研究的热点。随着人们对材料环境安 全性的关注, 新型无卤阻燃剂包括具有新型化学结 构的无卤阻燃剂 ${ }^{[4-5]}$ 、基于传统无卤阻燃剂的复合阻 燃剂 ${ }^{[6-7]}$ 及纳米阻燃剂不断被开发出来。纳米阻燃剂 具有添加量少、阻燃效率高等优点, 其中石墨烯 ${ }^{[8]}$ 、 碳纳米管 $^{[9]}$ 、层状蒙脱土 ${ }^{[10]}$ 等纳米阻燃剂得到了迅 速发展。近年来, 金属复盐如羟基锡酸盐(羊基锡酸 锌、羟基锡酸铁等)因其无毒环保和阻燃消烟性在阻 燃领域得到广泛关注 ${ }^{[11]}$ 。羟基锡酸盐还能够被调控 形成纳米尺度的立方体、球形或棒状 ${ }^{[12]}$, 是具有应 用前景的纳米阻燃剂。但由于无机纳米阻燃剂易团 聚, 且与树脂基体相容性差, 在实际应用中通常需 要用有机物对其进行表面改性 ${ }^{[13]}$ 。磷腈类衍生物作 为新型无卤阻燃剂, 具有环境友好、与聚合物相容 性高、阻燃性能优异等特性而备受关注 ${ }^{[14-15]}$ 。本课 题组赵师师等 ${ }^{[16]}$ 利用六氯环三磷腈( HCCP) 和 4, 4二羟基二苯砜(BPS)为原料, 合成了具有高度环交 联结构的聚环三磷腈-二羟基二苯砜(PZS)微球和微 纳米管, 并将其应用于阻燃领域, 结果表明 PZS 在 聚碳酸酯和环氧树脂中均起到良好的阻燃效果。

本工作采用均相沉淀法合成羟基锡酸锶 $\left(\mathrm{SrSn}(\mathrm{OH})_{6}\right)$ 纳米棒, 并用 PZS 对其进行包覆改性, 得到 PZS 包覆 $\mathrm{SrSn}(\mathrm{OH})_{6}$ 纳米棒 $\left(\mathrm{PZS} @ \mathrm{SrSn}(\mathrm{OH})_{6}\right)$, 进而考察 $\mathrm{SrSn}(\mathrm{OH})_{6}$ 及 PZS@ $@ \mathrm{SrSn}(\mathrm{OH})_{6}$ 对环氧树脂 阻燃效果的影响, 还对 PZS@SrSn $(\mathrm{OH})_{6}$ 的阻燃机理 进行了探讨。

\section{1 实验方法}

\section{$1.1 \operatorname{SrSn}(\mathrm{OH})_{6}$ 纳米棒的制备}

在 $25{ }^{\circ} \mathrm{C}$ 下, 将 $10 \mathrm{~mL} 0.1 \mathrm{~mol} / \mathrm{L}$ 的 $\mathrm{NaOH}$ 溶液 加入到 $50 \mathrm{~mL} 0.1 \mathrm{~mol} / \mathrm{L}$ 的 $\mathrm{Sr}\left(\mathrm{NO}_{3}\right)_{2}$ 溶液中, 再在快
速磁力搅拌条件下, 将 $50 \mathrm{~mL} 0.1 \mathrm{~mol} / \mathrm{L}$ 的 $\mathrm{Na}_{2} \mathrm{SnO}_{3}$ 水溶液缓慢滴加入上述水溶液中, 滴加完毕继续反 应 $1 \mathrm{~h}$ 将白色沉淀过滤、洗涤至中性, 在 $60{ }^{\circ} \mathrm{C}$ 真空 烘箱干燥 $12 \mathrm{~h}$, 得到差基锡酸锶纳米棒 $\left(\mathrm{SrSn}(\mathrm{OH})_{6}\right)$ 。

\subsection{PZS@SrSn $(\mathrm{OH})_{6}$ 的制备}

称取 $0.213 \mathrm{~g}$ BPS 溶解于 $87.5 \mathrm{~mL}$ 乙腈, 再加入 $10 \mathrm{~mL}$ 三乙胺(TEA)。将 $1 \mathrm{~g} \mathrm{SrSn}(\mathrm{OH})_{6}$ 分散于 $150 \mathrm{~mL}$ 乙醇中, 并将其与 BPS 乙腈溶液混合, 置于 超声波清洗器中。将 $0.095 \mathrm{~g} \mathrm{HCCP}$ 溶于 $87.5 \mathrm{~mL}$ 乙 腈中, 滴加到上述混合溶液中, 滴加时间为 $40 \mathrm{~min}$, 滴加完毕后超声反应 $6 \mathrm{~h}$, 反应温度 $50{ }^{\circ} \mathrm{C}$, 超声功 率 $100 \mathrm{~W}$ 。反应产物在真空 $60{ }^{\circ} \mathrm{C}$ 下干燥至恒重, 得 到 PZS@SrSn $(\mathrm{OH})_{6}$, 反应过程如图 1 所示。

\section{$1.3 \mathrm{EP}$ 阻燃复合材料的制备}

称取 $50 \mathrm{~g}$ EP 置于雉形瓶中, 分别加入质量比 为 $1 \% 、 2 \% 、 3 \%$ 的 $\mathrm{SrSn}(\mathrm{OH})_{6}$ 和 $\mathrm{PZS} @ \mathrm{SrSn}(\mathrm{OH})_{6}$, 摚 拌 $30 \mathrm{~min}$, 再加入 $5.5 \mathrm{~g}$ 间苯二胺, 搅拌 $20 \mathrm{~min}$ 。之 后在模具中浇铸, 真空 $60{ }^{\circ} \mathrm{C}$ 下脱气 $20 \mathrm{~min}, 80{ }^{\circ} \mathrm{C}$ 固 化 $2 \mathrm{~h}, 150{ }^{\circ} \mathrm{C}$ 继续固化 $3 \mathrm{~h}$, 得到阻燃样条。

\section{4 性能表征}

TENSOR-27 型傅里叶变换红外光谱仪(FT-IR), $\mathrm{KBr}$ 压片，扫描步频 $2 \mathrm{~cm}^{-1}$, 扫描范围 4000 $400 \mathrm{~cm}^{-1}$, Bruker 公司; STA449CQMS403C 型热重分 析仪(TG), $\mathrm{N}_{2}$ 氛围, 升温速率 $10{ }^{\circ} \mathrm{C} / \mathrm{min}$, 测试温度 区间 35 800 ${ }^{\circ} \mathrm{C}$, 耐驰公司; TM-3000 型扫描电子显 微镜(SEM), 日本 JEOL 公司; Tecnai G2 F20 STWIN 型透射电子显微镜(TEM), FEI 公司; HC-3 oxygen index meter 型极限氧指数测试仪(LOI), 依 照国标 GB 2406-1993 进行测试, 南京江宁分析仪器 有限公司; D8 ADVANCE 型 $\mathrm{X}$ 射线粉末衍射仪 (XRD), 德国布鲁克仪器有限公司; Phenom ProX 型 能谱仪(EDS), 荷兰 Phenom-World 公司; EN iCone Plus 型锥形量热仪 (CONE), 样品尺寸 $10 \mathrm{~cm} \times$ $10 \mathrm{~cm} \times 3 \mathrm{~mm}$, 热辐照功率 $50 \mathrm{~kW} / \mathrm{m}^{2}$, 每个样品进 行 3 次平行实验取平均值, 英国 FTT 公司。 


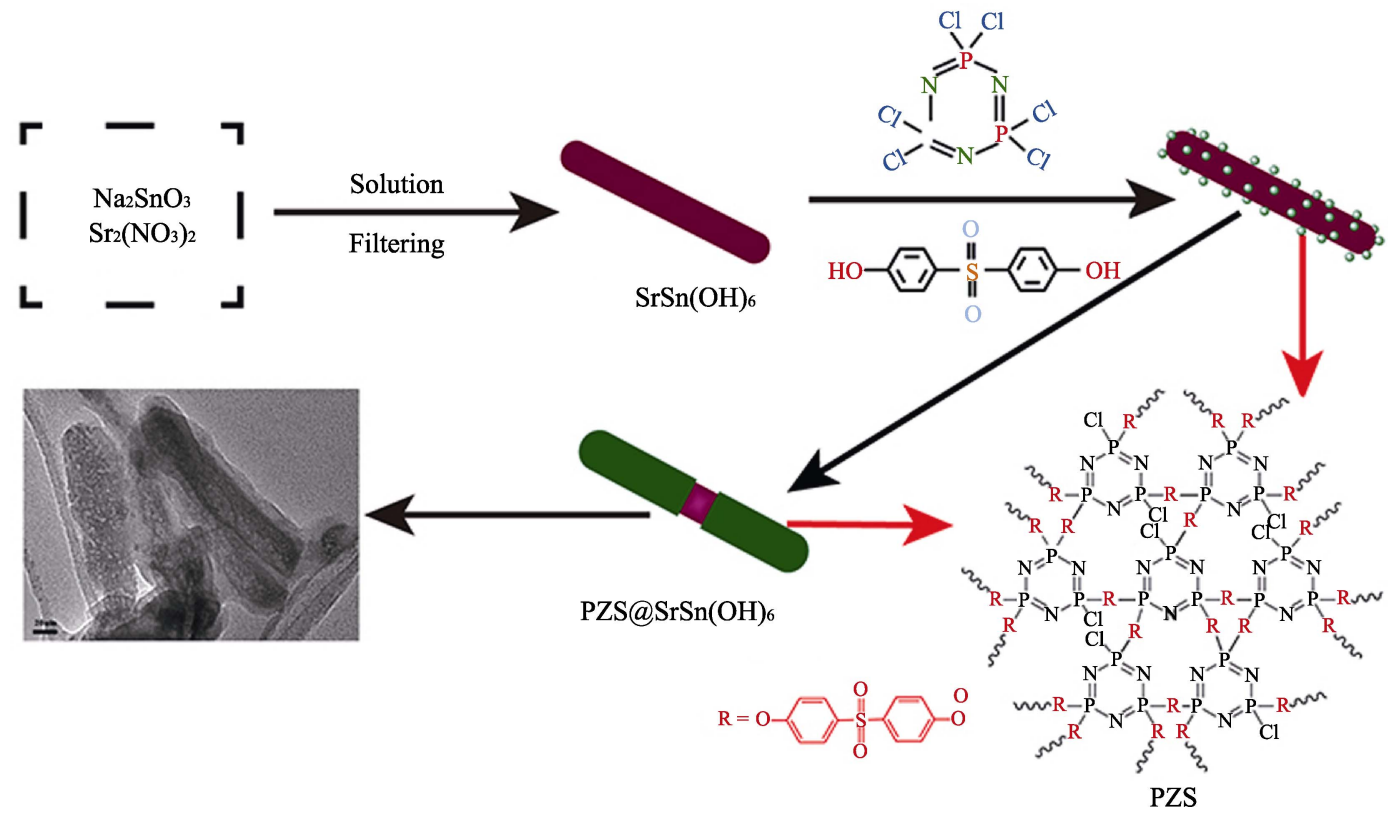

图 $1 \mathrm{SrSn}(\mathrm{OH})_{6}$ 及 $\mathrm{PZS} @ \mathrm{SrSn}(\mathrm{OH})_{6}$ 的合成路线图

Fig. 1 Synthetic routes of the $\mathrm{SrSn}(\mathrm{OH})_{6}$ and PZS@ $\mathrm{SrSn}(\mathrm{OH})_{6}$

\section{2 结果与讨论}

\section{1 $\operatorname{SrSn}(\mathrm{OH})_{6}$ 和 PZS $@ \operatorname{SrSn}(\mathrm{OH})_{6}$ 纳米棒的表征}

图 2(a, c) 分别为 $\mathrm{SrSn}(\mathrm{OH})_{6}$ 及 $\mathrm{PZS} @ \mathrm{SrSn}(\mathrm{OH})_{6}$ 纳米棒的微观形貌。从中可以看出, 两者均呈棒状, 其中 $\operatorname{SrSn}(\mathrm{OH})_{6}$ 平均直径 $112.8 \mathrm{~nm}$, 包覆后直径 $128.9 \mathrm{~nm}$ ，纳米棒表面平整光滑; 而 PZS@SrSn $(\mathrm{OH})_{6}$
表面较为粗粘, 边缘模糊。TEM 结果(图 2(b, d)) 显 示 PZS@ $\mathrm{SrSn}(\mathrm{OH})_{6}$ 具有明显的核壳结构, PZS 包覆 层厚度约为 $30 \mathrm{~nm}$ 。从图 2(e)的元素面分布图可以 看出, PZS@SrSn $(\mathrm{OH})_{6}$ 含有 $\mathrm{Sn} 、 \mathrm{Sr} 、 \mathrm{~S} 、 \mathrm{P}$ 等元素，其 中 $\mathrm{Sn} 、 \mathrm{Sr}$ 仅分布于核区域，而 $\mathrm{S} 、 \mathrm{P}$ 元素在整个核 壳范围均匀分布, 说明缩聚反应后 PZS 成功包覆在 $\mathrm{SrSn}(\mathrm{OH})_{6}$ 纳米棒的表面。
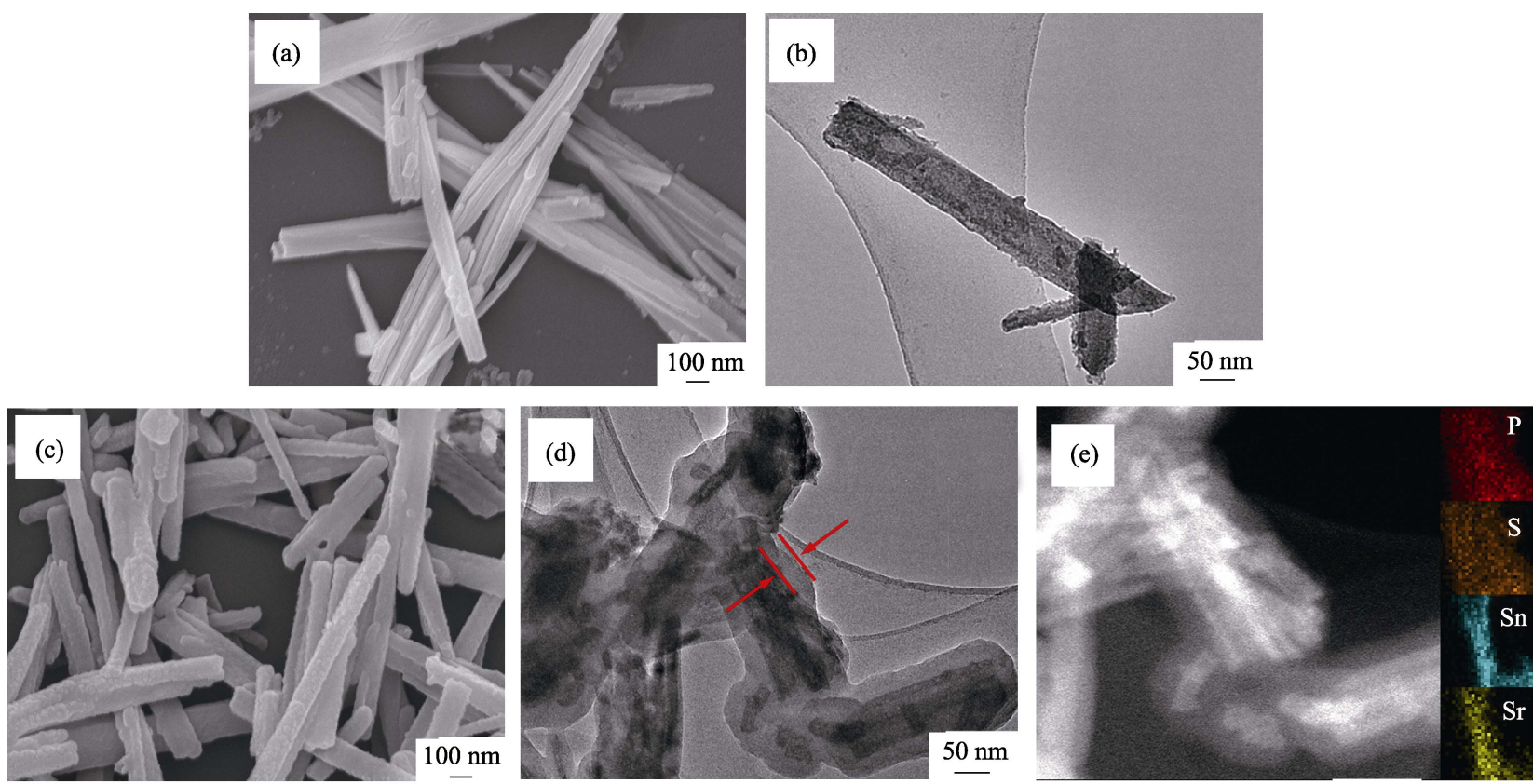

图 $2 \mathrm{SrSn}(\mathrm{OH})_{6}$ 及 $\mathrm{PZS} @ \mathrm{SrSn}(\mathrm{OH})_{6}$ 的微观形貌及其元素分布图

Fig. 2 Morphologies and element distribution map of $\operatorname{SrSn}(\mathrm{OH})_{6}$ and PZS@SrSn $(\mathrm{OH})_{6}$ $(\mathrm{a}, \mathrm{b}) \mathrm{SrSn}(\mathrm{OH})_{6}$; (c, d, e) PZS@ $\mathrm{SrSn}(\mathrm{OH})_{6}$ 


\section{2 阻燃 EP 复合材料的热降解性能}

阻燃剂热解过程及其成炭量是评判其阻燃效 果和选择阻燃聚合物类型及加工条件的重要依 据 ${ }^{[17-18]}$ 。图 3 和图 4 是 $\mathrm{SrSn}(\mathrm{OH})_{6} 、 \mathrm{PZS}$ 、 $\mathrm{PZS} @ \mathrm{SrSn}(\mathrm{OH})_{6}$ 和环氧树脂复合材料的热降解曲 线。由图 3 可知, $\mathrm{SrSn}(\mathrm{OH})_{6}$ 在 $217{ }^{\circ} \mathrm{C}$ 时开始失重, 此 阶段对应物理结合水的脱除; 在 $237^{\circ} \mathrm{C}$ 时达到最大 失重速率, $\mathrm{SrSn}(\mathrm{OH})_{6}$ 在此阶段转化成 $\mathrm{SrSnO}_{3}$; 最终 失重率为 $19.6 \mathrm{wt} \%$, 与理论失重率 $17.5 \mathrm{wt} \%$ 基本吻 合。 PZS 的 $T_{5 \%}$ 为 $442.8{ }^{\circ} \mathrm{C}$, 具有优异的热稳定性; PZS 热降解为一步降解, 当温度达到 $800{ }^{\circ} \mathrm{C}$ 时残炭 率达 $48.1 \%$, 说明 PZS 具有较高的成炭能力。 PZS@SrSn $(\mathrm{OH})_{6}$ 呈现三步降解，前两个阶段分别对 应 $\mathrm{SrSn}(\mathrm{OH})_{6}$ 和 $\mathrm{PZS}$ 的热降解过程, 第三阶段是由 于 $\mathrm{SrSnO}_{3}$ 的存在, $\mathrm{PZS}$ 进一步交联成炭的过程。对 比第一阶段 $\mathrm{SrSn}(\mathrm{OH})_{6}$ 降解后的残炭率, 计算可知 PZS@ $\operatorname{SrSn}(\mathrm{OH})_{6}$ 中 PZS 的质量分数约为 $30 \%$ 。值得 注意的是, PZS 对 $\mathrm{SrSn}(\mathrm{OH})_{6}$ 包覆后, PZS 和 $\mathrm{SrSn}(\mathrm{OH})_{6}$ 的最大热降解速率均大幅降低。

从图 4 及表 1 可知, $\mathrm{SrSn}(\mathrm{OH})_{6}$ 的加入使 $\mathrm{EP}$ 的 $T_{5 \%}$ 和 $T_{\text {max }}$ 由原来的 $364 、 375{ }^{\circ} \mathrm{C}$ 分别降低到 354 、 $367{ }^{\circ} \mathrm{C}$, 说明 $\mathrm{SrSn}(\mathrm{OH})_{6}$ 对 $\mathrm{EP}$ 有催化降解效应。而 加入 PZS@SrSn $(\mathrm{OH})_{6}$ 后, $T_{5 \%}$ 和 $T_{\max }$ 分别降低到 360
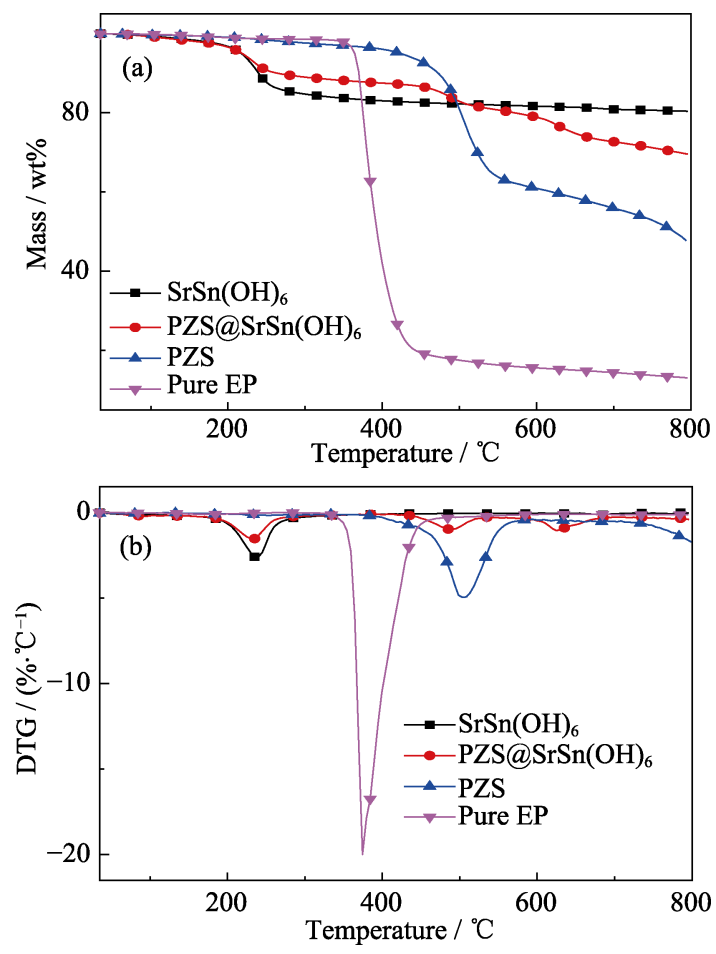

图 $3 \mathrm{PZS}, \mathrm{SrSn}(\mathrm{OH})_{6}$ 和 $\mathrm{PZS} @ \operatorname{SrSn}(\mathrm{OH})_{6}$ 在 $\mathrm{N}_{2}$ 氛围中测得 的热失重曲线( $(a)$ 和失重微分曲线 $(b)$

Fig. 3 TG (a) and DTG (b) curves of $\operatorname{SrSn}(\mathrm{OH})_{6}$, PZS and PZS@SrSn $(\mathrm{OH})_{6}$ tested in $\mathrm{N}_{2}$ atmosphere
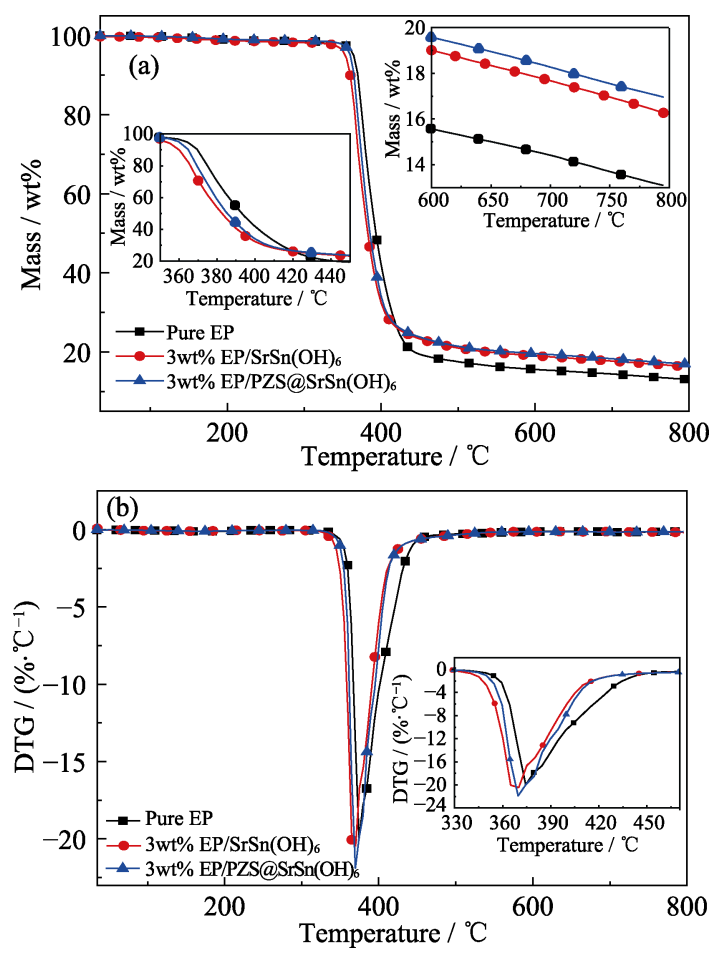

图 4 EP 纳米复合材料在 $\mathrm{N}_{2}$ 氛围中测得的热失重曲线(a)和 失重微分曲线(b)

Fig. 4 TG (a) and DTG (b) curves of EP nanocomposites tested in $\mathrm{N}_{2}$ atmosphere

\section{表 1 PZS, $\operatorname{SrSn}(\mathrm{OH})_{6}, \mathrm{PZS} @ \operatorname{SrSn}(\mathrm{OH})_{6}$} 及其 EP 纳米复合材料的热降解数据

Table 1 Thermogravimetric properties of $\mathrm{PZS}, \operatorname{SrSn}(\mathrm{OH})_{6}$, PZS@SrSn $(\mathrm{OH})_{6}$ and EP nanocomposites

\begin{tabular}{ccccc}
\hline Sample & & & $\begin{array}{c}\text { Residue } \\
\text { at }\end{array}$ & $\begin{array}{c}\text { Theoreti- } \\
\text { cal resi- } \\
\text { due at }\end{array}$ \\
& & & $\begin{array}{c}{ }^{\circ} \mathrm{C} \\
800{ }^{\circ} \mathrm{C} \%\end{array}$ & $\begin{array}{c}800{ }^{\circ} \mathrm{C} \\
/ \mathrm{wt} \%\end{array}$ \\
\hline $\mathrm{PZS}$ & 442.8 & 507.9 & 48.2 & - \\
$\mathrm{SrSn}(\mathrm{OH})_{6}$ & 217.3 & 237.7 & 80.4 & - \\
$\mathrm{PZS} @ \mathrm{SrSn}(\mathrm{OH})_{6}$ & 218.5 & 487.5 & 69.4 & - \\
$\mathrm{Pure} \mathrm{EP}$ & 364.2 & 375.3 & 13.0 & - \\
$3 \% \mathrm{EP} / \mathrm{SrSn}(\mathrm{OH})_{6}$ & 354.0 & 367.3 & 16.2 & 15.0 \\
$3 \% \mathrm{EP} / \mathrm{PZS} @ \mathrm{SrSn}(\mathrm{OH})_{6}$ & 360.0 & 368.6 & 17.0 & 14.7 \\
\hline
\end{tabular}

和 $368{ }^{\circ} \mathrm{C}$ 。 $\mathrm{EP}$ 的最大失重温度区间为 $330 \sim 450{ }^{\circ} \mathrm{C}$, $\mathrm{SrSn}(\mathrm{OH})_{6}$ 和 $\mathrm{PZS} @ \mathrm{SrSn}(\mathrm{OH})_{6}$ 的加入使最大失重区 间进一步缩小。 $800{ }^{\circ} \mathrm{C}$ 时 $\mathrm{EP}$ 复合材料的残炭率随 $\mathrm{SrSn}(\mathrm{OH})_{6}$ 和 $\mathrm{PZS} @ \mathrm{SrSn}(\mathrm{OH})_{6}$ 的加入提高至 16.2\% 和 $17.0 \%$, 高于理论值 $15.0 \%$ 和 $14.7 \%$ (理论值根据 简单混合比和各自失重率计算得出), 说明加入 $\mathrm{SrSn}(\mathrm{OH})_{6}$ 能够促进环氧树脂提前催化成炭。热重 分析结果表明 $\mathrm{PZS}$ 与 $\mathrm{SrSn}(\mathrm{OH})_{6}$ 可以协同催化 $\mathrm{EP}$ 的交联成炭。

\section{3 阻燃 EP 复合材料的阻燃性能}

极限氧指数(LOI) 是一种常用的高分子材料燃 
烧性能表征手段。添加阻燃剂前后环氧树脂复合材 料的 LOI 值如图 5 所示，由图得知，随着 $\mathrm{SrSn}(\mathrm{OH})_{6}$ 和 PZS@SrSn $(\mathrm{OH})_{6}$ 阻燃剂含量的增加, EP 复合材料 的 LOI 值得到显著提高。在添加相同量阻燃剂的情 况下, EP/PZS@ $@ \mathrm{SrSn}(\mathrm{OH})_{6}$ 复合材料的 LOI 值比 $\mathrm{EP} / \mathrm{SrSn}(\mathrm{OH})_{6}$ 高出约 $1 \%$ 。当仅添加 $3 \mathrm{wt} \%$ 的 PZS@ $\mathrm{SrSn}(\mathrm{OH})_{6}$ 时, LOI 值从纯 EP 的 26.2\%增加到 $29.6 \%$ 。通过观察 LOI 测试后的残炭宏观形貌，可以 看出 $\mathrm{EP} / \mathrm{PZS} @ \mathrm{SrSn}(\mathrm{OH})_{6}$ 的残炭量明显高于 $\mathrm{EP} / \mathrm{SrSn}(\mathrm{OH})_{6}$, 说明 $\mathrm{PZS} @ \mathrm{SrSn}(\mathrm{OH})_{6}$ 可以更有效地 提高 EP 的阻燃性能。

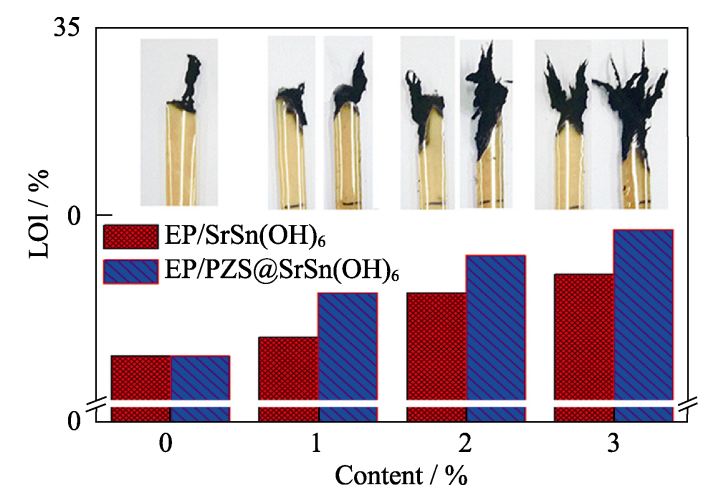

图 $5 \mathrm{EP}$ 及其纳米复合材料的极限氧指数及测试后残炭形貌 Fig. 5 LOI values and residue chars at the end of LOI tests of $\mathrm{EP}, \mathrm{EP} / \mathrm{SrSn}(\mathrm{OH})_{6}$ and EP/PZS@SrSn $(\mathrm{OH})_{6}$ nanocomposites
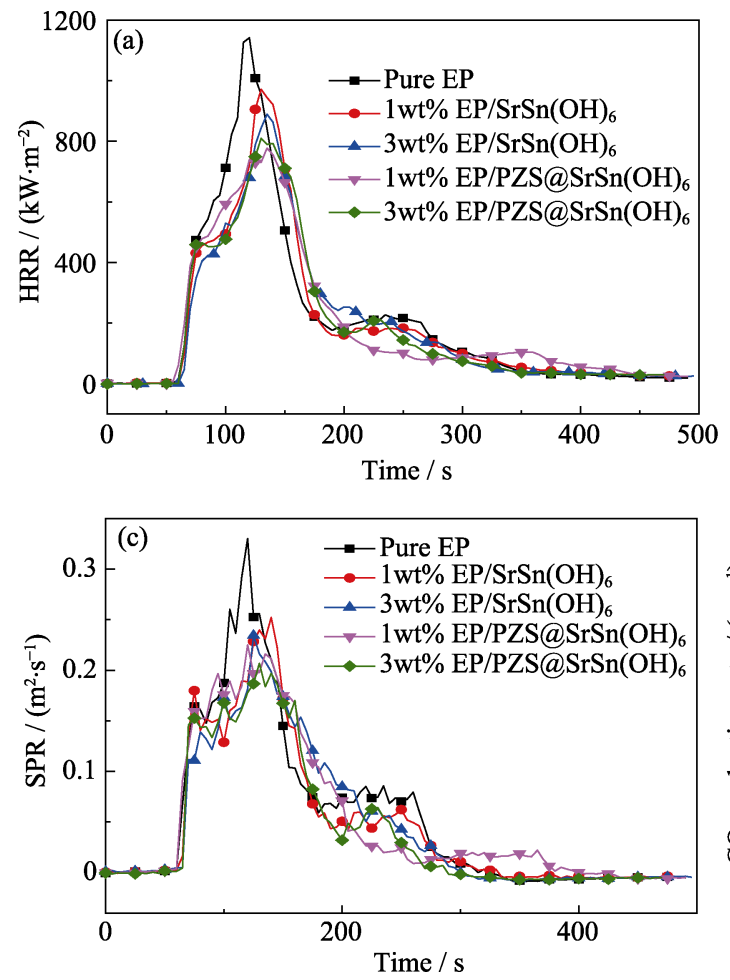

为进一步研究 EP 纳米复合材料的燃烧性能, 对阻燃前后的样品进行了雉形量热测试, 结果如图 6 和表 2 所示, 添加 $3 \mathrm{wt} \% \mathrm{SrSn}(\mathrm{OH})_{6}$ 和PZS@SrSn $(\mathrm{OH})_{6}$ 后, EP 复合材料残炭量均有大幅提高, 由纯 $\mathrm{EP}$ 的 $5.6 \%$ 分别增大到 $12.4 \%$ 和 $19.2 \%$, 增长幅度为 $126 \%$ 和 $242 \%$, 说明 $\mathrm{SrSn}(\mathrm{OH})_{6}$ 和 $\mathrm{PZS} @ \mathrm{SrSn}(\mathrm{OH})_{6}$ 可以显著 促进环氧树脂成炭, 且 $\mathrm{PZS} @ \mathrm{SrSn}(\mathrm{OH})_{6}$ 成炭效果更 强。从图 6(a) 可以看出, 纯 EP 的热释放和烟释放曲线 峰形尖锐, 加入 $3 \mathrm{wt} \% \mathrm{SrSn}(\mathrm{OH})_{6}$ 后, 热释放速率峰值 (PHRR) 和烟释放速率峰值(PSPR)由 $1141.1 \mathrm{~kW} / \mathrm{m}^{2}$ 和 $0.33 \mathrm{~m}^{2} / \mathrm{s}$ 降低到 $888.9 \mathrm{~kW} / \mathrm{m}^{2}$ 和 $0.24 \mathrm{~m}^{2} / \mathrm{s}$ 。而加入 $3 \mathrm{wt} \%$ PZS@SrSn $(\mathrm{OH})_{6}$ 后, PHRR 和 PSPR 进一步下 降到 $809.7 \mathrm{~kW} / \mathrm{m}^{2}$ 和 $0.21 \mathrm{~m}^{2} / \mathrm{s}$, 分别降低了约 $29 \%$ 和 $37 \%$ 。热释放总量也由 $100.4 \mathrm{MJ} / \mathrm{m}^{2}$ 下降到 92.6 和 $88.9 \mathrm{MJ} / \mathrm{m}^{2}$ 。生烟速率 $(\mathrm{SPR})$ 曲线(图 6(c)) 与 $\mathrm{CO}$ 速率曲线(图 6(d)) 与 HRR 类似, 且 PZS@ $\mathrm{SrSn}(\mathrm{OH})_{6}$ 对 SPR 和 CO 的抑制效果更为显著。加入 $3 \mathrm{wt} \%$ PZS@ $\mathrm{SrSn}(\mathrm{OH})_{6}$ 后, 生烟总量(TSP)也下降到 23.9 和 $21.2 \mathrm{~m}^{2}$, 说明 $\mathrm{SrSn}(\mathrm{OH})_{6}$ 和 $\mathrm{PZS} @ \mathrm{SrSn}(\mathrm{OH})_{6}$ 有良 好的抑烟性。对比之下可看出 PZS@SrSn $(\mathrm{OH})_{6}$ 对 EP 的阻燃效果更为显著, 说明 PZS 对 $\mathrm{SrSn}(\mathrm{OH})_{6}$ 的 包覆使两者之间具有协同阻燃效应。

\section{4 阻燃 EP 复合材料的残炭分析}

$\mathrm{EP}$ 纳米复合材料锥形量热后的残炭宏观形貌
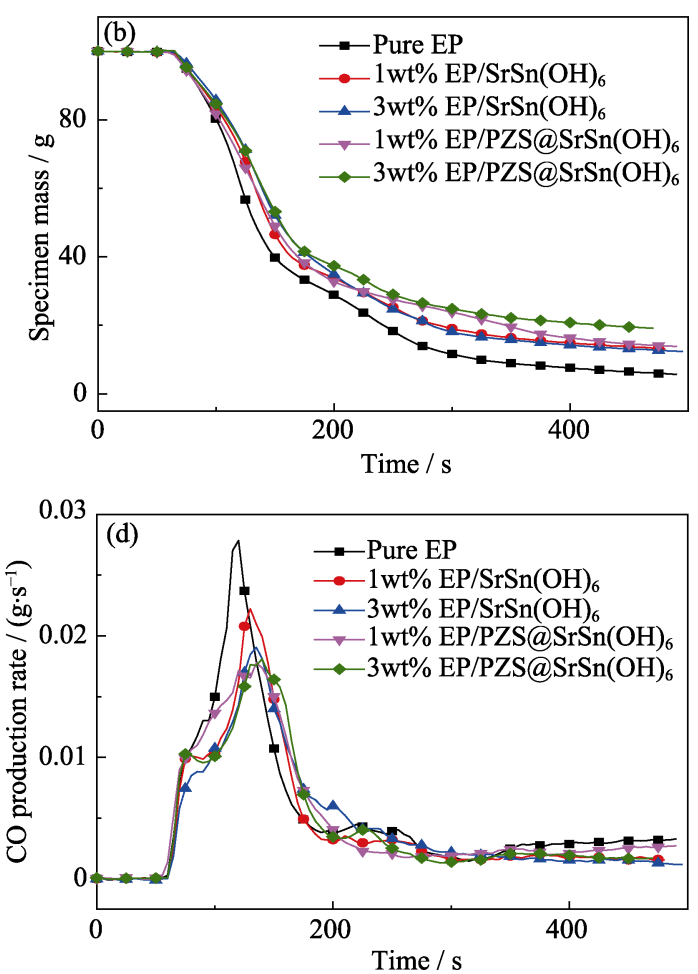

图 $6 \mathrm{EP}$ 及其纳米复合材料的热释放速率 (a), 质量损失 (b), 烟释放速率 (c) 和 CO 释放速率 $(\mathrm{d})$

Fig. 6 Heat release rate (HRR) (a), specimen mass (b), smoke production rate SPR (c), and $\mathrm{CO}$ production rate $(\mathrm{d})$ curves of the samples in CONE tests 
表 $2 \mathrm{EP}$ 及其纳米复合材料的锥形量热数据

Table 2 Cone calorimetry data for EP composites

\begin{tabular}{cccccc}
\hline Sample & Pure EP & $\begin{array}{c}1 \mathrm{wt} \% \\
\mathrm{EP} / \mathrm{SrSn}(\mathrm{OH})_{6}\end{array}$ & $\begin{array}{c}3 \mathrm{wt} \% \\
\mathrm{EP} / \mathrm{SrSn}(\mathrm{OH})_{6}\end{array}$ & $\begin{array}{c}1 \mathrm{wt} \% \\
\mathrm{EP} / \mathrm{PZS} @ \operatorname{SrSn}(\mathrm{OH})_{6}\end{array}$ & $\begin{array}{c}3 \mathrm{wt} \% \\
\mathrm{EP} / \mathrm{PZS} @ \mathrm{SrSn}(\mathrm{OH})_{6}\end{array}$ \\
\hline Char residue $/ \mathrm{g}$ & 5.6 & 13.2 & 12.4 & 13.8 & 19.2 \\
$\mathrm{THR} /\left(\mathrm{MJ} \cdot \mathrm{m}^{-2}\right)$ & 100.4 & 93.2 & 92.6 & 93.1 & 88.9 \\
$\mathrm{PHRR} /\left(\mathrm{kW} \cdot \mathrm{m}^{-2}\right)$ & 1141.1 & 971.7 & 888.9 & 777.3 & 809.7 \\
$\mathrm{PSPR} /\left(\mathrm{m}^{2} \cdot \mathrm{s}\right)$ & 0.33 & 0.25 & 0.24 & 0.22 & 0.21 \\
$\mathrm{TSP} / \mathrm{m}^{2}$ & 27.6 & 23.7 & 23.9 & 25.1 & 21.2 \\
\hline
\end{tabular}

如图 7 所示, 可以看出纯 EP 燃烧后只剩极少量松散 的残炭。加入 $\mathrm{SrSn}(\mathrm{OH})_{6}$ 和 $\mathrm{PZS} @ \mathrm{SrSn}(\mathrm{OH})_{6}$ 后, 残 炭厚度和残炭量均有大幅提高，且残炭更加完整。 $\mathrm{EP} / \mathrm{PZS} @ \mathrm{SrSn}(\mathrm{OH})_{6}$ 残炭颜色稍浅, 质感更加厚重 坚韧。致密且厚实的炭层有助于保护 EP 基体和阻 隔热质传递，从而提高材料的阻燃性。

为了进一步研究阻燃机制, 用 XRD、FT-IR 和 EDS 对雉形量热后的残炭微观结构进行分析, 如 图 8 所示, 由图得知, 燃烧后 $\mathrm{SrSn}(\mathrm{OH})_{6}$ 纳米棒转化 为 $\mathrm{SrSnO}_{3}, \mathrm{SrSn}(\mathrm{OH})_{6}$ 受热失水降低体系温度和可 燃气体的浓度, 生成的 $\mathrm{SrSnO}_{3}$ 进一步隔热隔氧, 从
而提高阻然效果。与添加 $\mathrm{EP} / \mathrm{SrSn}(\mathrm{OH})_{6}$ 相比, $\mathrm{PZS} @ \mathrm{SrSn}(\mathrm{OH})_{6}$ 的残炭中形成了 $\mathrm{Sr}_{2} \mathrm{SnO}_{4}$ 。在此过 程中, PZS 的降解形成含磷的焦炭层可进一步促进 基体与可燃小分子的交联成炭，这种密集骨架型的 炭层结构进一步增大炭层厚度并提高残炭的阻隔能 力。残炭的能谱图和红外光谱(图 8(b, c)) 显示 1271、 1027 和 $1110 \mathrm{~cm}^{-1}$ 处 $\mathrm{P}=\mathrm{O}$ 键和 $\mathrm{P}-\mathrm{O}-\mathrm{C}$ 键的形成, 说 明 PZS 在热降解过程中形成了磷酸或偏磷酸类物 质。而 PZS 降解过程中形成的磷酸和偏磷酸类物质 可以促使 EP 树脂基体交联成炭，进一步提高阻燃 效果。
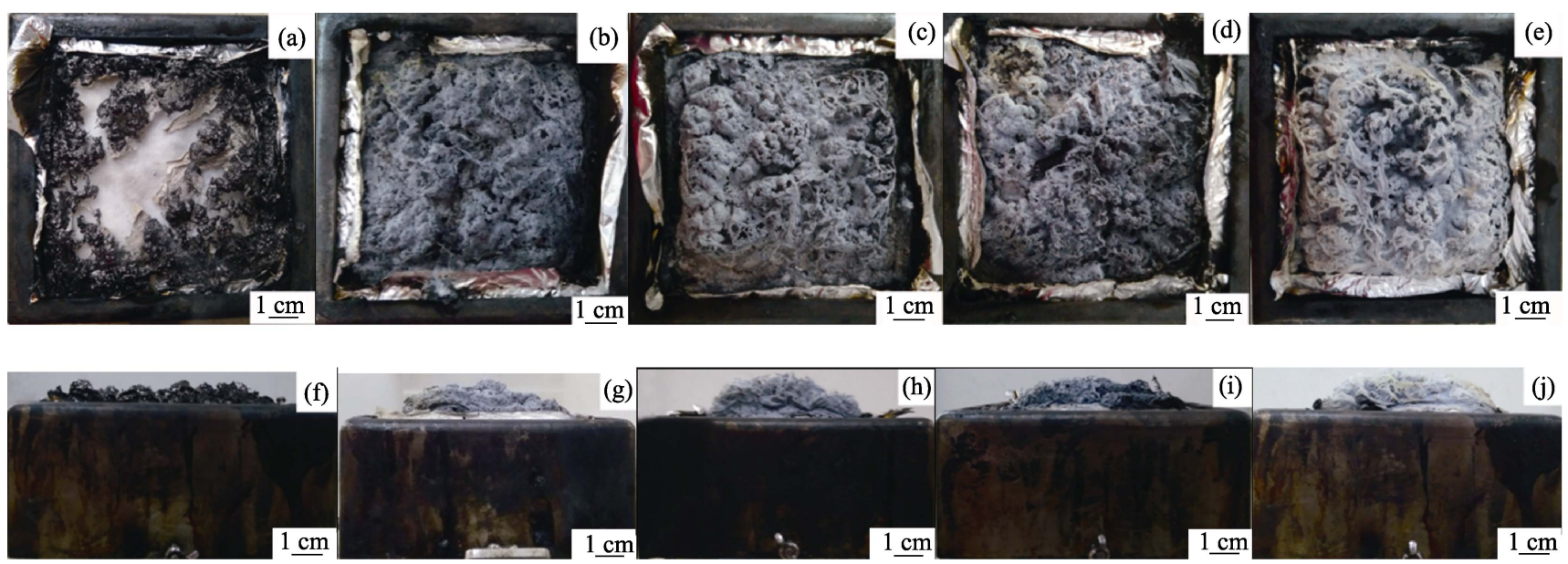

图 $7 \mathrm{EP}$ 及其纳米复合材料雉形量热后残炭宏观形貌

Fig. 7 Digital photos of the char residues for composites after cone calorimeter tests (a,f) EP; (b,g) 1wt\% EP/SrSn $(\mathrm{OH})_{6} ;$ (c,h)3wt\% EP/SrSn $(\mathrm{OH})_{6}$; (d,i) 1wt\% EP/PZS@SrSn(OH) 6 ; (e,j) 3wt\% EP/PZS@SrSn $(\mathrm{OH})_{6}$
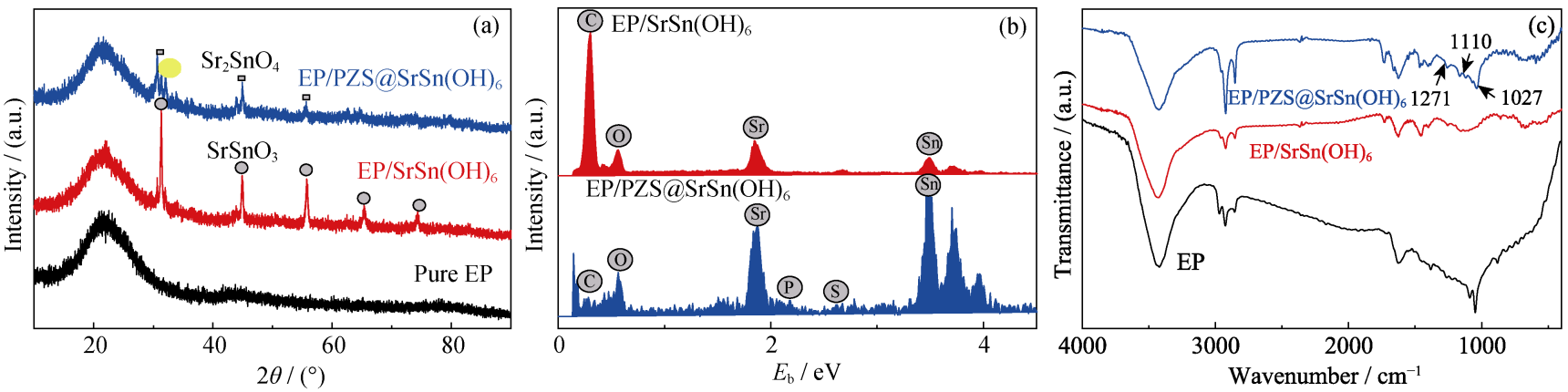

图 8 EP 及其纳米复合材料残炭的 XRD(a), EDS(b)及 FTIR(c)图谱

Fig. 8 XRD (a), EDS (b) and FT-IR (c) spectra of the char residue of EP samples 


\section{3 结论}

通过均相沉淀法合成了一种 $\operatorname{SrSn}(\mathrm{OH})_{6}$ 纳米棒 阻燃剂, 并用环交联磷腈衍生物(PZS)对其进行表 面 包覆 功能化，得 到 有机无 机杂化 的 $\mathrm{PZS} @ \mathrm{SrSn}(\mathrm{OH})_{6}$ 纳米阻燃剂, 并将其成功应用于环 氧树脂的阻燃。PZS@SrSn $(\mathrm{OH})_{6}$ 是一种优异的抑烟 成炭剂, 可以显著提高 EP 的阻燃性能。当 $\mathrm{PZS} @ \mathrm{SrSn}(\mathrm{OH})_{6}$ 添加量为 $3 \mathrm{wt} \%$ 时，阻然 EP 复合材 料的极限氧指数从纯 EP 的 $26.2 \%$ 提高到了 $29.6 \%$, 燃烧过程中热释放速率、总热释放、总烟释放以及 烟释放速率均显著降低，且燃烧后形成致密的纳米 纤维网络结构炭层。PZS@SrSn $(\mathrm{OH})_{6}$ 对环氧树脂是 一种优良、高效，具有潜在应用价值的阻燃剂。本 研究对具有特殊形貌锡酸盐阻燃剂的合成及阻燃功 能化应用具有一定的指导意义和参考价值。

\section{参考文献:}

[1] SHAO Z B, ZHANG M X, LI Y, et al. A novel multi-functional polymeric curing agent: synthesis, characterization, and its epoxy resin with simultaneous excellent flame retardance and transparency. Chem. Eng. J., 2018, 345: 471-482.

[2] QIAN L J, QIU Y, WANG J Y, et al. High-performance flame retardancy by char-cage hindering and free radical quenching effects in epoxy thermosets. Polymer, 2015, 68: 262-269.

[3] JIAN X Y, HE Y, LI Y D, et al. Curing of epoxidized soybean oil with crystalline oligomeric poly(butylene succinate) towards high performance and sustainable epoxy resins. Chem. Eng. J., 2017, 326: $875-885$.

[4] WANG P, XIA L, JIAN R K, et al. Flame-retarding epoxy resin with an efficient $\mathrm{P} / \mathrm{N} / \mathrm{S}$-containing flame retardant: preparation, thermal stability, and flame retardance. Polym. Degrad. Stabil., 2018, 149: 69-77.

[5] CHEN T, CHEN X M, WANG M J, et al. A novel halogen-free co-curing agent with linear multi-aromatic rigid structure as flameretardant modifier in epoxy resin. Polym. Advan. Technol., 2018, 29(1): 603-611.

[6] XIE H L, LAI X J, LI H Q, et al. Fabrication of ZrP nanosheet decorated macromolecular charring agent and its efficient synergism with ammonium polyphosphate in flame-retarding polypro- pylene. Compos. Part A-Appl. S., 2018, 105: 223-234.

[7] YANG Y Y, LIU J, CAI X F. Antagonistic flame retardancy between hexakis(4-nitrophenoxy) cyclotriphosphazene and potassium diphenylsulfone sulfonate in the PC system. J. Therm. Anal. Calorim., 2016, 126(2): 571-583.

[8] SHI X X, PENG X F, ZHU J Y, et al. Synthesis of DOPO-HQ-functionalized graphene oxide as a novel and efficient flame retardant and its application on polylactic acid: thermal property, flame retardancy, and mechanical performance. J. Colloid. Interf. Sci., 2018, 524: 267-278.

[9] YANG W, TAWIAH B, YU C, et al. Manufacturing, mechanical and flame retardant properties of poly(lactic acid) biocomposites based on calcium magnesium phytate and carbon nanotubes. Compos. Part A-Appl. S., 2018, 110: 227-236.

[10] CHEN G G, HU Y J, PENG F, et al. Fabrication of strong nanocomposite films with renewable forestry waste/montmorillonite/ reduction of graphene oxide for fire retardant. Chem. Eng. J., 2018, 337: 436-445.

[11] ZHANG B, LIU H, HAN J. Synthesis of zinc hydroxystannate microcapsule for improving flame retardancy and smoke suppression of poly(lactic acid). Mater. Lett., 2018, 213: 35-39.

[12] XU R, DENG B, MIN L, et al. $\mathrm{CuSn}(\mathrm{OH})_{6}$ submicrospheres: room-temperature synthesis and weak antiferromagnetic behavior. Mater. Lett., 2011, 65(4): 733-735.

[13] GAO T T, SANG B, SHAO B, et al. Flame retardancy and mechanical properties of a novel zinc hydroxystannate/epoxy resin nanocomposite. J. Nanosci. Nanotechno., 2017, 17(12): 8856-8863.

[14] QIU S L, MA C, WANG X, et al. Melamine-containing polyphosphazene wrapped ammonium polyphosphate: a novel multifunctional organic-inorganic hybrid flame retardant. J. Hazard. Mater, 2018, 344: 839-848.

[15] YANG R, WANG B, HAN X F, et al. Synthesis and characterization of flame retardant rigid polyurethane foam based on a reactive flame retardant containing phosphazene and cyclophosphonate. Polym. Degrad. Stabil., 2017, 144: 62-69.

[16] ZHAO S S, HE M, SONG W Y, et al. Synthesis of a phosphorus/ nitrogen/sulphur containing phosphazene micro-nanotube and its flame retardancy on epoxy nanocomposite. Chem. J. Chinese U., 2017, 38 (12): 2337-2343

[17] LI P, FU H, ZHAO O, et al. Influence of polyphosphate flame retardant couple with ammonium polyphosphate on epoxy resin. Chem. J. Chinese U., 2017, 38(2): 294-302.

[18] WEN P Y, WANG X F, XING W Y, et al. Synthesis of a novel triazinebased hyperbranched char foaming agent and the study of its enhancement on flame retardancy and thermal stability of polypropylene. Ind. Eng. Chem. Res., 2013, 52(48): 17015-17022. 